\title{
Home Versus Hospital Intravenous Antibiotic Therapy for Acute Pulmonary Exacerbations in Children With Cystic Fibrosis
}

\begin{abstract}
Dena Nazer, MD, ${ }^{1}$ Ibrahim Abdulhamid, $\mathrm{MD}^{2 \star}{ }^{2 \star}$ Ronald Thomas, $\mathrm{PhD},{ }^{3}$ and Sara Pendleton, $\mathrm{MD}^{4}$
Summary. To compare the effectiveness of home versus hospital intravenous (IV) antibiotic therapy for acute pulmonary exacerbations in children with cystic fibrosis (CF). A retrospective chart review was performed of 143 encounters for pulmonary exacerbations in 50 patients with CF. All encounters were categorized into two groups based on location of completion of antibiotic therapy: hospital group completed treatment in hospital $(n=64)$, home group completed treatment at home $(n=79)$. Percent change was calculated for forced vital capacity (FVC), forced expiratory volume in $1 \mathrm{sec}$ (FEV1), forced expiratory flow rate between 25 percent and 75 percent of vital capacity $\left(\mathrm{FEF}_{25-75 \%}\right)$, maximum forced expiratory flow $\left(\mathrm{FEF}_{\mathrm{max}}\right)$, oxygen saturation (O2 SAT), and weight. Means of percent change (PC) from the beginning to the end of IV antibiotic treatment in outcome variables were compared. Total duration of treatment was compared between the two groups. The two groups had no significant differences at baseline in all outcome variables. Treatment of exacerbations in both groups resulted in significant improvement of lung function, $\mathrm{O} 2$ SATS, and weight $(P \leq 0.001)$. The percent change in FEV1 was greater in hospital group versus home group $(P=0.04)$. The duration of treatment was significantly shorter in the hospital group $(P=0.001)$. Home and hospital IV antibiotic therapy resulted in significant improvement in lung function and weight. Hospital therapy, however, resulted in significantly greater improvement in FEV-1 and required less duration of treatment as compared to home treatment in children with CF. Pediatr Pulmonol. 2006; 41:744-749. ๑ 2006 Wiley-Liss, Inc.
\end{abstract}

Key words: cystic fibrosis; intravenous antibiotics; pulmonary exacerbations; children.

\section{INTRODUCTION}

The basic genetic defect in CF causes chronic airway infection with several bacterial pathogens especially Gram negative organisms such as Pseudomonas Aeroginosa. ${ }^{1-3}$ This infection leads to bronchiectasis, progressive loss of lung function, and respiratory failure. Affected patients commonly develop recurrent pulmonary exacerbations with symptoms of increased coughing, sputum production, fever, weight loss, and acute deterioration of lung function. ${ }^{4}$ Oral and IV antibiotics given to treat acute pulmonary exacerbations result in subjective and objective improvement of symptoms, lung function, improvement in the quality of life (QOL), and longer life expectancy in patients with $\mathrm{CF}^{4}$

In children and adults with $\mathrm{CF}$, several weeks of IV antibiotic courses, intense airway clearance, chest physiotherapy, and nutritional support have been the main therapeutic interventions for severe pulmonary exacerbations in North America. ${ }^{4}$ The alternative European approach in children and adults with CF prefers regular intermittent IV antibiotic courses even in the absence of acute respiratory symptoms. ${ }^{5,6}$ Over the last 40 years, many different treatment strategies have been implemented including antibiotics administered at the home and hospital which led to improved survival of adults and children with $\mathrm{CF}^{5}$ Since the introduction of home IV antibiotic treatment, many investigators have reported

${ }^{1}$ Department of Pediatric Education, Children's Hospital of Michigan, Wayne State University, Detroit, Michigan.

${ }^{2}$ The Carman and Ann Adams Department of Pediatrics, Division of Pulmonary Medicine, Children's Hospital of Michigan, Wayne State University, Detroit, Michigan.

${ }^{3}$ Children's Research Center of Michigan, Children's Hospital of Michigan, Wayne State University, Detroit, Michigan

${ }^{4}$ The Carman and Ann Adams Department of Pediatrics, Children's Hospital of Michigan, Wayne State University, Detroit, Michigan.

Presented in part at the 17th Annual North American Cystic Fibrosis Conference.

*Correspondence to: Ibrahim Abdulhamid, M.D., Children's Hospital of Michigan, 3901 Beaubien Boulevard, Detroit, MI. 48201.

E-mail: ihamid@med.wayne.edu

Received 5 October 2005; Revised 28 November 2005; Accepted 16 February 2006

DOI 10.1002/ppul.20433

Published online 15 June 2006 in Wiley InterScience

(www.interscience.wiley.com). 
their experiences and comparisons of home to hospital IV antibiotic treatments of CF lung disease. ${ }^{7-20}$ These studies involved adult patients or groups of adults and children.

Home IV antibiotic treatment is an attractive alternative because of its potential benefits that may include reducing frequency of hospital admissions, decrease hospital stay, increased independence from the hospital, earlier intervention, less risk of cross infection, better QOL, and more cost savings. ${ }^{20}$ However, three recent studies disputed the effectiveness of routine use of home IV antibiotics for patients with $\mathrm{CF}$ with minimum supervision. ${ }^{15-17}$ The cost effectiveness and effect on QOL of home treatment of pulmonary exacerbations were also shown to be less favorable than hospital treatment by two other studies. ${ }^{18,19}$

Most of the studies that compared hospital versus home IV antibiotic treatment interventions included either adult patients only or had a significant number of adult subjects enrolled; none was carried out exclusively in the pediatric age group. The impact of the location of treatment could be different on children with $\mathrm{CF}$ and their families as compared to adult patients.

We conducted this retrospective study to compare the effectiveness of home versus hospital IV antibiotics in the treatment of acute pulmonary exacerbations in children with $\mathrm{CF}$ in an urban tertiary center under regular practice conditions.

\section{MATERIALS AND METHODS}

\section{Study Design}

The design of this study was a retrospective chart review. We reviewed charts of children with $\mathrm{CF}$ admitted to Children's Hospital of Michigan between the years of 1994 and 2003. The diagnosis of CF was based on a documented abnormal sweat chloride testing, genotyping, and typical clinical signs and symptoms.

\section{Inclusion and Exclusion Criteria}

We included patients between 6 and 21 years of age with an acute pulmonary exacerbation requiring IV antibiotics. We defined an acute pulmonary exacerbation clinically as appearance or exaggeration of several symptoms including: dyspnea, fever, fatigue, increased cough, increased volume and changed appearance of sputum, decreased appetite, and/or weight loss. ${ }^{4}$ Pulmonary function tests (PFT), O2 SAT, and weights were documented at the onset and after completion of the IV antibiotic course.

We excluded from our study patients younger than 6 years of age due to their inability to perform a reliable PFT. We also excluded patients' encounters with the following criteria: FEV1 less than $25 \%$ predicted, severe pulmonary or other CF-related complication such as: pneumothorax, massive hemoptysis, hematemesis, intestinal obstruction, liver failure, or significant weight loss requiring gastrostomy tube placement at the time of IV antibiotic therapy. Charts of all children who met our inclusion/exclusion criteria were reviewed.

\section{Treatment Protocol}

Each pulmonary exacerbation requiring IV antibiotics was considered an encounter. Encounters were subsequently divided into two groups based on location of completion of IV antibiotics. All treatments were initiated in the hospital. The hospital group was defined as those patients whose IV antibiotics were given at the hospital entirely. The home group was defined as those patients who completed IV antibiotics at home after a maximum of 8 days of hospital IV antibiotics. Patients in the home group were then discharged from the hospital for completion of IV antibiotics, other medications, nutrition, and chest physiotherapy at home.

In both groups, antibiotics were chosen based on sputum culture and antibiotic sensitivity results done at the University Laboratories at Detroit Medical Center/Wayne State University. Most patients received a combination of an aminoglycoside and broad-spectrum penicillins, or third generation cephalosporins. In the hospital group a multidisciplinary team was involved in treatment. It included the medical staff, nurses, respiratory therapists, social workers, and dieticians. In the home group, parents and trained family members were instructed on how to administer IV antibiotics and chest physiotherapy by the durable medical equipment (DME) suppliers, hospital based IV team, and the CF team. Medications were mostly administered by parents and caregivers of children in the home group. Older children helped with medication administration, nebulized treatments, and chest physiotherapy under supervision. All of our patients lived with their caregivers; none of our patients lived alone. Patients were also advised to optimize nutrition and monitor improvement of symptoms. Patients had to be in a relatively stable respiratory state on discharge from the hospital.

\section{Location of Treatment}

Decision on location of treatment was based on patients' and caregivers' preferences, physician's approval, available resources, and prior experience of patients and their caregivers. Caregivers selecting home treatment had to be willing to provide home therapy, available, adherent to treatment, and easily contacted.

\section{Treatment Follow-Up}

In the both groups aminoglycoside levels, blood urea nitrogen (BUN) and creatinine serum levels were 
measured during the initial hospitalization and doses of aminoglycosides were adjusted accordingly. The BUN and creatinine serum levels were measured once or twice per week during the treatment period. In the home group, blood was drawn at home and results were sent to the treating physician. Our CF team nurses did not provide any home visits for the home group. However, patients had access to the $\mathrm{CF}$ nurse coordinator and the $\mathrm{CF}$ team physicians on a 24-hr basis for any questions, concerns, or IV access. DME personnel were also available for questions or concerns with the home equipment, medications, supplies, and problems with IV access.

Patients from the home group were seen at 1- to 2-week intervals throughout their treatment and more frequently if symptoms indicated. Immediately at the end of antibiotic treatment (within 1-2 days), patients were scheduled for a clinic visit. During that visit, patients underwent a clinical evaluation and PFT, which helped to make the decision whether to continue treatment with IV antibiotics, or not.

\section{Outcome Variables}

PFT, O2 SAT, and weights were documented at the onset and after completion of the IV antibiotic course. PFT parameters included FEV1, FVC, $\mathrm{FEF}_{25-75}$, and $\mathrm{FEF}_{\max }$.

Primary outcome variables were FEV1 and total duration of therapy with IV antibiotics. Secondary outcome variables were $\mathrm{FVC}, \mathrm{FEF}_{25-75 \%}, \mathrm{FEF}_{\max }$, O2 SAT, and weight. The two groups were divided into three categories according to the severity of FEV1 at the time of initiation of intravenous antibiotics (T1). Patients from each group were classified as mild when FEV1 was more than $70 \%$, moderate when FEV1 was between $40 \%$ and $69 \%$, and severe when FEV1 was between 39 and $25 \%$.

\section{Data Analysis}

An independent samples $t$-test was employed to examine mean differences at baseline with continuously scaled variables. A Fisher's exact test was selected for comparisons between study groups on categorically scaled variables. An analysis-of-covariance (ANCOVA) examined mean differences in the primary outcomes of FEV1 and length of treatment between the two study groups while controlling for baseline values. Statistically significant differences were considered achieved at a $P$ value $\leq 0.05$, two-tailed. Appropriate assumptions for mean comparisons (normality and homogeneity of variance) were checked and verified. If violated, nonparametric tests (i.e., Mann-Whitney $U$, Friedman) were substituted.

Time 1 (T1) was defined as the outcome variable at the time of initiation of therapy and time 2 (T2) was defined as the value after completion of antibiotic course. Percent change $[\mathrm{PC}=(\mathrm{T} 2-\mathrm{T} 1) / \mathrm{T} 1 \times 100]$ was calculated for each outcome variable.
Secondary analyses examined mean differences in FEV1 and length of treatment holding severity outcome (mild, moderate, or severe) and then gender (male vs. female) as additional factor variables in a two-factor ANOVA. All statistical procedures were conducted using SPSS Version 13.0.

This study was approved by the Wayne State University Human Investigation Committee.

\section{RESULTS}

\section{Patients}

Twenty-seven patients had encounters in the hospital and 23 at home. We identified 143 encounters of pulmonary exacerbations in patients with CF, with 64 in the hospital group and 79 in the home group. The mean age of all patients was found to be $12.7 \pm 3.8$ years.

The majority of encounters were for Caucasian children (90.2\%), 9.1\% were for African Americans, and 0.7\% were for Hispanics. Female patients had $60.1 \%$ of the encounters while $39.9 \%$ of the encounters were for males.

No statistically significant differences were found between study groups at baseline relative to age, gender, weight, or PFT (Table 1).

Results of the two-factor ANOVA revealed no significant differences in mean FEV1 values between the two study groups when examined according to disease severity classification at treatment initiation (Table 2).

Treatment of exacerbations in both groups resulted in significant improvement of lung function, O2 SATS and weight $(P \leq 0.001)$.

Overall, FEV1 percent change (PC) was significantly higher in the hospital group $(39 \pm 3.7)$ compared to the home group $(23 \pm 30.0) \quad(P=0.04) \quad$ (Table 3$)$. Although not statistically significant mean changes in $\mathrm{FVC}, \mathrm{FEF}_{25-75}$, and $\mathrm{FEF}_{\max }$ were also higher for patients in the hospital group in comparison to those in the home group (Table 3 ).

TABLE 1-Comparison of Outcome Variables Between the Home and Hospital Groups at Baseline

\begin{tabular}{lccc}
\hline Variable & $\begin{array}{c}\text { Home group } \\
\text { mean (SD) }\end{array}$ & $\begin{array}{c}\text { Hospital group } \\
\text { mean (SD) }\end{array}$ & $P$-value \\
\hline Age & 13 years $(3.7)$ & 13 years $(3.9)$ & 0.29 \\
Weight $(\mathrm{kg})$ & $38(12.8)$ & $38(13.9)$ & 0.83 \\
FVC $_{\text {FEF }_{25-75}}$ & $69 \%(17.4)$ & $71 \%(13.8)$ & 0.36 \\
FEF $_{\max }$ & $34 \%(30.2)$ & $30 \%(16.8)$ & 0.24 \\
FEV1 & $51 \%(16.2)$ & $50 \%(21.9)$ & 0.78 \\
O2 SAT & $53 \%(17.1)$ & $52 \%(14.2)$ & 0.67 \\
\hline
\end{tabular}

FVC, forced vital capacity; $\mathrm{FEF}_{25-75}$, mean expiratory flow at $25-75 \%$ of FVC; FEF, maximum expiratory flow; FEV1, forced expiratory volume in $1 \mathrm{sec}$; $\mathrm{O} 2 \mathrm{SAT}$, oxygen saturation. All pulmonary function changes are listed as percent predicted. 
TABLE 2-Comparison of Disease Severity Between the Home and Hospital Groups at Treatment Initiation Based on FEV1 Percent Predicted Value

\begin{tabular}{lccc}
\hline $\begin{array}{l}\text { Severity of } \\
\text { pulmonary disease }\end{array}$ & $\begin{array}{c}\text { Home group } \\
\text { mean (SE) }\end{array}$ & $\begin{array}{c}\text { Hospital group } \\
\text { mean (SE) }\end{array}$ & $P$-value \\
\hline Mild & $83(2.1)$ & $78(3.0)$ & 0.22 \\
Moderate & $53(1.0)$ & $54(1.2)$ & 0.75 \\
Severe & $32(1.8)$ & $33(2.1)$ & 0.72 \\
\hline
\end{tabular}

$\mathrm{SD}$, standard deviation.

The duration of IV antibiotic therapy was significantly shorter in the hospital group $(16 \pm 5.0)$ than in the home group $(19 \pm 5.6)(P=0.001)$ (Table 3$)$.

The hospital group patients with severe lung disease had greater improvement in FEV1 $(93 \pm 9.4)$, in comparison to the home group $(45 \pm 7.8)(P=0.001)$ (Table 4$)$.

Although not statistically different, mean PC in FEV1 in both the mild and moderate severity categories was also higher in the hospital group than in the home group (Table 4). In the mild category, the hospital group recorded a mean FEV1 change of $7 \pm 8.3$ and in the home group $2 \pm 5.3(P=0.64)$. In the moderate category of severity, the hospital group recorded a mean FEV1 change of $29 \pm 3.4$ and the home group $21 \pm 3.1(P=0.12)$.

Mean length of treatment between the home and hospital study groups, when examined according to disease severity, revealed a significantly shorter length of treatment for patients with moderate lung disease in the hospital group $(16 \pm 0.8)$ than for patients in the home group $(19 \pm 0.8)(P=0.02)$. Mean length of treatment was also consistently shorter for patients in the hospital group even with mild and severe disease (Table 5).

Lastly, mean length of treatment in days within a gender type by study group differed significantly for females. Females in the home group recorded a higher mean treatment length $(19 \pm 0.8)$ in comparison to females in the hospital group $(15 \pm 0.9)(P=0.001)$. Although not

TABLE 3-Comparison of Percent Change Between the Home and Hospital Groups at the End of Treatment

\begin{tabular}{lccc}
\hline Variable & $\begin{array}{c}\text { Home group } \\
\text { mean (SD) }\end{array}$ & $\begin{array}{c}\text { Hospital group } \\
\text { mean (SD) }\end{array}$ & $P$-value \\
\hline $\begin{array}{l}\text { Length of treatment } \\
\quad \text { days) }\end{array}$ & $19(5.6)$ & $16(5.0)$ & 0.001 \\
FEV1 & $23(30.0)$ & $39(3.7)$ & 0.04 \\
FVC & $17(23.0)$ & $24(23.4)$ & 0.10 \\
FEF $_{25-75}$ & $45(62.8)$ & $67(85.5)$ & 0.21 \\
FEF $_{\text {max }}$ & $29(35.4)$ & $52(63.6)$ & 0.10 \\
O2 SAT & $2(2.3)$ & $1(2.3)$ & 0.53 \\
Weight $(\mathrm{kg})$ & $3(3.0)$ & $1(2.3)$ & 0.91 \\
\hline
\end{tabular}

FVC, forced vital capacity; $\mathrm{FEF}_{25-75}$, mean expiratory flow at $25-75 \%$ of FVC; FEF, maximum expiratory flow; FEV1, forced expiratory volume in $1 \mathrm{sec} ; \mathrm{O} 2 \mathrm{SAT}$, oxygen saturation; SD, standard deviation. All pulmonary function changes are listed as percent change form baseline.
TABLE 4-Comparison of Percent Change in FEV1 Based on Severity of Lung Disease in the Home and Hospital Groups

\begin{tabular}{lccc}
\hline Severity subgroup & $\begin{array}{c}\text { Home group } \\
\text { mean (SE) }\end{array}$ & $\begin{array}{c}\text { Hospital group } \\
\text { mean (SE) }\end{array}$ & $P$-value \\
\hline Mild & $2(5.3)$ & $7(8.3)$ & 0.64 \\
Moderate & $21(3.1)$ & $29(3.4)$ & 0.12 \\
Severe & $45(7.8)$ & $93(9.4)$ & 0.001 \\
\hline
\end{tabular}

FEV1, forced expiratory volume in $1 \mathrm{sec}$; SE, standard error.

statistically significant, mean length of treatment in days for males between study groups was slightly higher for males in the home group $(18 \pm 0.9)$ than for males in the hospital group $(17 \pm 1.0)$ (Table 6$)$.

\section{DISCUSSION}

Both home and hospital IV antibiotic treatments resulted in significant improvement from baseline in our patient population. However, hospital IV antibiotic courses lead to greater improvement in lung function and required a shorter duration of treatment than home IV antibiotic treatment.

Over the last 2 decades, studies from several countries have shown conflicting results concerning the benefits of home versus hospital IV antibiotic treatment of CF lung infections. ${ }^{7-20}$ The evaluated outcomes in these reports included changes in lung function, duration of treatment, QOL, anthropometric measures, and cost effectiveness. The majority of patients enrolled in these studies were adults. Comparing these studies could be problematic because of their differences in several aspects such as patients' age, outcome measures, study designs (prospective vs. retrospective; open vs. random enrollment), treatment indications, and length of treatment periods. In addition, the small number of subjects included in most of the studies may have prevented differences between hospital and home IV antibiotics from reaching statistical significance and possibly masking the superiority of one intervention versus the other. Various studies revealed that home IV antibiotic treatments had similar clinical outcomes, provided better QOL and were less costly than

TABLE 5-Comparison of Length of Treatment in Days Based on the Severity of Lung Disease in the Home and Hospital Groups

\begin{tabular}{lccc}
\hline Severity subgroup & $\begin{array}{c}\text { Home group } \\
\text { mean (SE) }\end{array}$ & $\begin{array}{c}\text { Hospital group } \\
\text { mean (SE) }\end{array}$ & $P$-value \\
\hline Mild & $16(1.5)$ & $13(2.2)$ & 0.14 \\
Moderate & $19(0.8)$ & $16(0.8)$ & 0.02 \\
Severe & $21(1.3)$ & $17(1.5)$ & 0.06 \\
\hline
\end{tabular}

SE, standard error. 
TABLE 6-Comparison of Length of Treatment in Days Between the Home and Hospital Groups Based on Gender Differences

\begin{tabular}{lccc}
\hline Gender & $\begin{array}{c}\text { Hospital group } \\
\text { mean (SE) }\end{array}$ & $\begin{array}{c}\text { Home group } \\
\text { mean (SE) }\end{array}$ & $P$-value \\
\hline Females & $15(0.9)$ & $19(0.8)$ & $<0.001$ \\
Males & $17(1.0)$ & $18(0.9)$ & 0.4 \\
\hline
\end{tabular}

SE, standard error.

hospital interventions ${ }^{8-14}$ in many European centers; patients with $\mathrm{CF}$ are given IV antibiotics on a regular basis even in the absence of acute pulmonary exacerbations as recommended by the European panel. ${ }^{5}$ Home treated patients are more carefully selected and better prepared for home care. In addition, many of the home treated subjects had visiting nurse support that assisted with administering the medications and chest physiotherapy.

This study is the first report that compared home to hospital IV antibiotic treatments exclusively in children with CF. Our results are in partial agreement with four recent reports that showed a better outcome of hospital IV antibiotics in patients with CF thought to be capable of performing this type of home care. ${ }^{15-18}$ Unlike Bosworth and Nielson, ${ }^{17}$ our results are similar to the findings reported by Bradley et al. ${ }^{16}$ that showed a significant increase in lung function in both hospital and home treated patients with better improvement in the hospital group. ${ }^{16}$ However, similar to Bosworth and Nielson ${ }^{17}$ the home treated group in our center required a longer duration of treatment in comparison to the hospital group. The results of our study and of other reports suggest that routine home IV antibiotic treatment of CF acute pulmonary exacerbations may be less beneficial.

We do not know the reasons for the superiority of hospital IV antibiotics in our pediatric patient population. We had no reason to question the adherence of our patients and their families. However, it is possible that a few of these children had missed some IV antibiotic doses, did not have vigorous chest physiotherapy, had insufficient rest, or inadequate nutritional support.

Although optimal adherence with home IV antibiotic administration was reported by Bosworth and Nielson, their patients reported less frequent and possibly less intense chest physiotherapy at home. ${ }^{17}$ Adherence with home therapy in adult patients with $\mathrm{CF}$ is variable and could conceivably be as low as $50 \% .{ }^{21}$ Possible reasons for improper adherence in adults with $\mathrm{CF}$ include inadequate adherence with IV therapy and airway clearance, and pressures to return early to school or work with less than adequate restful and recovery time. ${ }^{22,23}$ In a questionnaire given to 50 adult patients with $\mathrm{CF}$ with $85 \%$ response rate, $70 \%$ of the subjects admitted missing 5 days of antibiotic doses per course, $84 \%$ had drugs left over, and $45 \%$ threw some drugs away. The main reasons given for the lack of optimal adherence included problems with venous access, failure to get up on time, inability to fit all the treatments in the busy daily schedules, and lack of interest in the treatment. ${ }^{22}$ Although we had no reasons to suspect suboptimal adherence from our patients or their caregivers in carrying their respective duties, we had no objective measures to evaluate their adherence. In our experience, we never had any reports, concerns, or complaints from DME suppliers that patients had any extra medications they never used.

\section{Limitations}

There were several limitations of our study. Since this was a retrospective study, patients could not be randomized to either the hospital or home group. The power of our study was limited and the results should be interpreted with caution, based upon our small sample size. In addition the exclusion of severely affected patients and patients with complications may have affected our results. We did not objectively assess adherence of patients and caregivers in the home group in terms of administration of IV antibiotics, nutrition, or chest physiotherapy. Newer modalities of treatments that emerged during our 10-year study period may have favorably affected the outcomes of patients recently enrolled. However, we assume that patients were treated according to the standard of care at time of enrollment regardless of where they were treated for their acute exacerbation. The design of our study, mainly the fact that it was a retrospective study made it hard to assess the long-term outcomes' effectiveness of treatment interventions. Lastly we did not collect information on the QOL in our patients during these years. Therefore, we were not able to study the impact of pulmonary exacerbations and compare the effect of the two interventions on QOL in both groups.

In summary, we evaluated the results of hospital and home IV antibiotic treatments of acute pulmonary exacerbations in children with CF. Both therapies resulted in significant improvement in pulmonary function and weight gain. However, hospital based intervention led to a much greater improvement in lung function and required shorter duration than home IV antibiotic treatment.

Randomized, prospective, long-term studies with a larger sample size of patients are needed to compare hospital versus home treatment in patients with CF with acute pulmonary exacerbations.

\section{REFERENCES}

1. Ratjen F, Doring G. Cystic fibrosis. Lancet 2003;361:681-689.

2. FitzSimmons SC. The changing epidemiology of cystic fibrosis. J Pediatr 1993;122:1-9.

3. Orenstein DM, Winnie GB, Altman H. Cystic fibrosis: a 2002 update. J Pediatr 2002;140:156-164. 
4. Smith AL. Antibiotic therapy in cystic fibrosis: evaluation of clinical trials. J Pediatr 1986;108:866-870.

5. Doring G, Conway SP, Heijerman HG, Hodson ME, Hoiby N, Smyth A, Touw DJ. Antibiotic therapy against Pseudomonas aeruginosa in cystic fibrosis: a European consensus. Eur Respir J 2000;16:749-767.

6. Frederiksen B, Lanng S, Koch C, Hoiby N. Improved survival in the Danish center-treated cystic fibrosis patients: results of aggressive treatment. Pediatr Pulmonol 1996;21:153-158.

7. Strandvik B, Hjelte L, Widen B. Home intravenous antibiotic treatment in cystic fibrosis. Scand J Gastroenterol Suppl 1988; 143:119-120.

8. Strandvik B, Hjelte L, Malmborg AS, Widen B. Home intravenous antibiotic treatment of patients with cystic fibrosis. Acta Paediatr 1992;81:340-344.

9. Gilbert J, Robinson T, Littlewood JM. Home intravenous antibiotic treatment in cystic fibrosis. Arch Dis Child 1988;63: 512-517.

10. Pond MN, Newport M, Joanes D, Conway SP. Home versus hospital intravenous antibiotic therapy in the treatment of young adults with cystic fibrosis. Eur Respir J 1994;7:1640-1644.

11. Winter RJ, George RJ, Deacock SJ, Shee CD, Geddes DM. Selfadministered home intravenous antibiotic therapy in bronchiectasis and adult cystic fibrosis. Lancet 1984;1(8390):13381339.

12. Donati MA, Guenette G, Auerbach H. Prospective controlled study of home and hospital therapy of cystic fibrosis pulmonary disease. J Pediatr 1987;111:28-33.

13. Wolter JM, Bowler SD, Nolan PJ, McCormack JG. Home intravenous therapy in cystic fibrosis: a prospective randomized trial examining clinical, quality of life and cost aspects. Eur Respir J 1997;10:896-900.
14. Riethmueller J, Busch A, Damm V, Ziebach R, Stern M. Home and hospital antibiotic treatment prove similarly effective in cystic fibrosis. Infection 2002;30:387-391.

15. Thornton J, Elliott R, Tully MP, Dodd M, Webb AK. Long term clinical outcome of home and hospital intravenous antibiotic treatment in adults with cystic fibrosis. Thorax 2004;59:242246.

16. Bradley JM, Wallace ES, Elborn JS, Howard JL, McCoy MP. An audit of the effect of intravenous antibiotic treatment on spirometric measures of pulmonary function in cystic fibrosis. Ir J Med Sci 1999;168:25-28.

17. Bosworth DG, Nielson DW. Effectiveness of home versus hospital care in the routine treatment of cystic fibrosis. Pediatr Pulmonol 1997;24:42-47.

18. Thornton J, Elliott RA, Tully MP, Dodd M, Webb AK. Clinical and economic choices in the treatment of respiratory infections in cystic fibrosis: Comparing hospital and home care. J Cyst Fibros 2005;4:239-247.

19. Yi MS, Tsevat J, Wilmott RW, Kotagal UR, Britto MT. The impact of treatment of pulmonary exacerbations on the healthrelated quality of life of patients with cystic fibrosis: does hospitalization make a difference? J Pediatr 2004;144:711718.

20. Van Aalderen WM, Mannes GP, Bosma ES, Roorda RJ, Heymans HS. Home care in cystic fibrosis patients. Eur Respir J 1995; $8: 172-175$.

21. Abbot J, Dodd M, Bilton D, Webb AK. Treatment compliance in adults with cystic fibrosis. Thorax 1994;49:115-120.

22. Phillips AM. Home intravenous antibiotic therapy: practical aspects in adults. J R Soc Med 1997;90(Suppl 31):34-36.

23. Fong S, Dales RE, Tierney MG. Compliance among adults with cystic fibrosis. DLCP Ann Pharmacother 1990;24:689-692. 